and cancer incidence. Personal air styrene measurements and biological markers of styrene exposure from the 1960s up to the present day will be used to update the exposure assessment. Linear mixed-effects models will be applied to develop a quantitative, historical, industry-specific job-exposure matrix. Predictors available in the individual cohorts include country, occupation, employment year, product, process, and task. Some cohorts will be pooled together prior to analysis, others analyzed separately following a common protocol that will focus on different exposure metrics (cumulative, duration, mean, highest attained, peaks) and exposure time windows. The aggregated data will be synthesized by a meta-analysis.

Results A total of 96,000 workers employed between 19472007 in 762 companies in Finland, Italy, UK, US, and Denmark, and over 2.8 million person years will be included, and 40,000 air samples and 13,000 urinary samples are identified. Conclusion Pooling and meta-analysis of existing cohorts are powerful tools in the search for more definite answers to the carcinogenicity of this important chemical.

\section{P-323 THE EFFECT OF THE ONSET OF A DISEASE ON EXIT FROM PAID EMPLOYMENT AMONG WORKERS IN THE NETHERLANDS: A LONGITUDINAL REGISTER-BASED STUDY WITH 11 YEARS FOLLOW-UP}

${ }^{1}$ Roos Hijdra, Suzan Robroek, Lex Burdorf, Merel Schuring. 'Erasmus Medical Center, Netherlands

\subsection{6/OEM-2021-EPI.270}

Introduction With increasing retirement age, employed persons are more likely to suffer from chronic diseases, such as cardiovascular diseases, diabetes, inflammatory diseases, respiratory diseases, and psychological disorders, at later stage during their working career.

Objectives This study investigates the influence of the onset of a disease on the likelihood of exit from paid employment through different pathways (unemployment, disability benefits, economic inactivity, early retirement).

Methods For this longitudinal study with 11 years of followup (2009-2019), 4,276,770 persons were selected, who were employed between 2009 and 2010, and used no medication for the selected diseases in 2009. Register data from Statistics Netherlands on medication use, employment status, and sociodemographic factors were used. Exit from paid employment pathways were defined as: unemployment, disability benefits, economic inactivity, and early retirement. The following six disease categories were identified based on medication: cardiovascular diseases, diabetes mellitus, respiratory illness, psychological disorders, inflammatory disorders and psychotic disorders. Descriptive statistics and Cox Proportional Hazards analyses with competing risks were performed.

Results The onset of any disease increased the likelihood of exit from paid employment, with strongest effect observed for psychological disorders (Hazard Ratio $[\mathrm{HR}]=1.37$, 95\% confidence interval $[\mathrm{CI}]$ 1.34-1.40) and psychotic disorders $(\mathrm{HR}=1.49,95 \%$ CI 1.41-1.58). Of all pathways out of paid employment, onset of a disease had the strongest effect on disability benefits, with HRs ranging from 1.75 (inflammatory diseases) to 9.30 (psychotic disorders).

Conclusion For the working age population, the onset of common disorders, especially psychological and psychotic disorders, is a risk for maintaining paid employment. Interventions are needed to prevent persons with these diseases from involuntary loss of paid employment.

\section{P-328 AGE DIFFERENCES IN WORK-DISABILITY DURATION ACROSS CANADA: EXAMINING VARIATIONS BY FOLLOW-UP TIME AND CONTEXT}

'Jonathan Fan, Robert Macpherson, Peter Smith, Anne M Harris, Monique Gignac, Chris McLeod. 'University of British Columbia, Canada

\subsection{6/OEM-2021-EPI.271}

Introduction Research has examined age-related patterns in return to work and wage-replacement duration following a workplace injury. The various clinical, functional or physiological factors studied do not fully account for age differences in wage-replacement duration. One contextual factor that has been largely overlooked in research studies is the potential impact of the phase of recovery.

Objectives This study aimed to understand age differences in wage-replacement duration by focusing on variations in the relationship across different periods of follow-up time.

Methods We used administrative claims data provided by six workers' compensation systems in Canada, focusing on timeloss claims for workers aged 15-80 years with a work-related injury/illness during the 2011 to 2015 period. Survival analysis examined age-related differences in the hazard of transitioning off (versus remaining on) disability benefits, allowing for relaxed proportionality constraints on the hazard rates over time. Differences were examined on the absolute (hazard difference) and relative (hazard ratios [HR]) scales.

Results Older age groups had a lower likelihood of transitioning off wage-replacement benefits compared to younger age groups in the overall models (e.g., 55-64 vs. 15-24 years: HR 0.62). However, absolute and relative differences in agespecific hazard rates varied as a function of follow-up time. The greatest age-related differences were observed at earlier event times and were attenuated towards a null difference across later follow-up times.

Conclusion Our study provides insight into the workplace injury/illness claim and recovery processes and suggests that older age is not always strongly associated with worse disability duration outcomes at longer disability durations. The use of data from multiple jurisdictions lends external validity to our findings and demonstrates the utility of using cross-jurisdictional data extracts. Future work should examine the social and contextual determinants that operate during various recovery phases, and how these factors interact with age.

\section{P-330 AGE DIFFERENCES IN RETURN-TO-WORK FOLLOWING INJURY: UNDERSTANDING THE ROLE OF AGE ACROSS LONGITUDINAL FOLLOW-UP}

'Jonathan Fan, Monique Gignac, Anne M Harris, Peter Smith. ${ }^{1}$ University of British Columbia, Canada

\subsection{6/OEM-2021-EPI.272}

Introduction Older age tends to be associated with longer time to return-to-work (RTW) following a workplace injury and multiple recurrences of work absence following an initial RTW attempt. However, few studies have examined the underlying factors that are responsible for these differences. 
Objectives To examine the overall association between age and return-to-work (RTW), understand the extent to which functional, psychosocial, organizational, life-stage related factors indirectly explain these associations, and examine whether there is a remaining direct proportion not mediated by these factors.

Methods We used survey data from a prospective cohort of injured workers in Victoria, Australia. Participants were recruited during the 2014 to 2015 period from monthly samples of claimants identified by the compensation system. Path models examined the relationship between age and RTW, and the proportion mediated via functional, psychosocial, organizational, life-stage related factors.

Results Older age was associated with non-RTW, although the pattern was not observed consistently across follow-up surveys. A proportion of the overall relationship between age and nonRTW was explained by functional and life-stage factors and RTW status at previous time points.

Conclusion Findings underscore the importance of moving beyond age measured only in chronological years, towards more complex conceptual and analytical models that recognize age as a multidimensional construct.

\section{P-333 USING CANJEM TO EXAMINE THE ASSOCIATION BETWEEN OCCUPATIONAL EXPOSURE TO SELECTED METALS, METALLOIDS, AND WELDING FUMES AND BRAIN CANCER IN THE INTEROCC POOLED INTERNATIONAL CASE-CONTROL STUDY}

Romain Pasquet, Jack Siemiatycki, Lesley Richardson, Elisabeth Cardis, Anita Koushik
${ }^{1}$ Centre de recherche du Centre hospitalier de I'Université de Montréal (CRCHUM), Canada

\subsection{6/OEM-2021-EPI.273}

Introduction Exposure to metallic compounds may contribute to the etiology of brain cancer; however, few epidemiologic studies have examined this potential association.

Objective To examine occupational exposure to 21 metallic compounds in relation to the risk of glioma and meningioma.

Methods INTEROCC is an international consortium of seven brain cancer case-control studies using a common protocol. Among 1,917 glioma cases, 1,827 meningioma cases, and 5,475 controls in the pooled INTEROCC population, job histories were collected and transformed into histories of exposure to 21 metallic compounds by linkage to the Canadian job-exposure-matrix. Three metrics of exposure were calculated for each agent: ever exposed, duration of exposure, and cumulative exposure. Conditional logistic regression was used to estimate the odds ratios (ORs) and their 95\% confidence intervals (95\% CIs) for the association between the three metrics of exposure and both glioma and meningioma.

Results There was no evidence of associations between our selected agents and glioma. There were positive associations, with ORs ranging from 1.20 to 2.40 , between meningioma and several of the metallic compounds, most notably zinc compounds, lead fumes, chromium VI compounds, soldering fumes, metal oxide fumes, and soldering fumes. Overall, our results were similar to two previous studies based on INTEROCC that examined five of the metallic compounds included in this study, using a modified version of the Finish job-exposure-matrix.
Conclusion Our results are suggestive of positive associations between exposure to metallic compounds, particularly metallic fumes, and meningioma, but not glioma.

\section{P-334 OCCUPATIONAL EXPOSURES EXPERIENCED BY MONTRÉAL WOMEN PARTICIPATING IN TWO CASE- CONTROL STUDIES}

${ }^{1}$ Romain Pasquet, Emmanuelle Batisse, France Labrèche, Mark Goldberg, Vikki Ho. 'Centre de recherche du Centre hospitalier de I'Université de Montréal (CRCHUM), Canada

\subsection{6/OEM-2021-EPI.274}

Introduction Women constitute nearly half of the workforce. However, most of our knowledge about occupational diseases come from studies conducted on men.

Objective To describe occupational exposures experienced by women.

Methods Two case-control studies of postmenopausal breast cancer were conducted in Montreal in 1996 and in 2011. Questionnaires on lifetime occupational history were administered during in-person or telephone interviews. Experts reviewed subjects' work history, assessing exposure to a list of 258 chemicals. Chemicals that were deemed to be present were categorized by concentration ('low', 'medium', 'high'), where low represented a background occupational level and high was the highest level experienced in that work environment. We pooled exposure information from both studies by time period and by age of exposure.

Results In both studies combined, the three most prevalent exposures were cleaning agents, ozone, and organic solvents; the jobs in which these top 3 agents were present included nurses and waitresses (cleaning agents), secretaries and clerks (ozone), and housekeepers and elementary school teachers (organic solvents). For cleaning agents and ozone, most exposures occurred at a low concentration (>98\%) while slightly higher exposures to organic solvents were found (14\% medium and $2 \%$ high). The top 3 agents by time period were: <1950, fabric dust, aliphatic aldehydes, and cotton dust; 1950-1969, cleaning agents, aliphatic aldehydes, and organic solvents; 1970-1989, cleaning agents, ozone, and aliphatic alcohols; and $\geq 1990$, ozone, cleaning agents and aliphatic alcohols. The prevalence of exposures differed for women exposed earlier versus later in their working life (predominant agents $\leq 35$ years of age: cleaning agents, aliphatic aldehydes, aliphatic alcohols; $>35$ years of age were ozone, cleaning agents, organic solvents).

Conclusion Occupational exposures of women remain understudied; bringing out inconspicuous exposures can help better assess women's occupational risks.

\section{P-336 GENDER DIFFERENCES IN OCCUPATIONAL EXPOSURE IN THE CANADIAN JOB-EXPOSURE-MATRIX (CANJEM)}

${ }^{1}$ Romain Pasquet, Jérôme Lavoué, Jack Siemiatycki, Marie-Elise Parent, France Labrèche, Mark Goldberg, Vikki Ho. 'Centre de recherche du Centre hospitalier de I'Université de Montréal (CRCHUM), Canada

\subsection{6/OEM-2021-EPI.275}

Introduction One of the principal challenges in communitybased occupational studies is retrospective assessment of exposure. Job-exposure-matrices (JEMs) have been proposed as a 